\title{
Pharmacokinetic and Toxicological Profile of Artemisinin Compounds: An Update
}

\author{
Bikash Medhi Sazal Patyar Ramya S. Rao Prasad Byrav DS Ajay Prakash \\ Department of Pharmacology, Postgraduate Institute of Medical Education and Research, Chandigarh, India
}

\section{Key Words}

Artemisinin - Malaria $\cdot$ Pharmacokinetics $\cdot$ Toxicokinetics

\begin{abstract}
Artemisinin has been used effectively in malaria treatment. With the emerging resistance to malaria, the optimum and judicial use of the drug has become important. The drug metabolism and toxicology can have an impact on the therapeutic profile and clinical use of this antimalarial agent. In this review, we discuss the pharmacokinetics and toxicological aspects of artemisinin and its therapeutic implications. Artemisinins have several dosing routes including oral, intramuscular, intravenous and rectal. With repeated dosing, artemisinin has propensity for autoinduction, leading to decreased plasma levels on repeated dosing. Combination with other antimalarials in most cases did not influence the pharmacokinetics of artemisinins. Interactions with cytochrome $\mathrm{P}_{450}$ inhibitors are known but these neither affect the efficacy nor the toxicity of the respective derivative. Artemisinins are generally regarded to be of low toxicity. Two major problems associated with them are neurotoxicity and reproductive toxicity. But the extent of this neurotoxicity is dependent on the nature of the compound, on the route of administration, and on the nature of the formulation. Moreover, it occurs in humans at very high doses. However, as a matter of precaution, the use of artemisinins in the first trimester of pregnancy has been contraindicated.
\end{abstract}

Copyright $\odot 2009$ S. Karger AG, Basel

\section{Introduction}

Artemisinin, earlier known as Qinghaosu, is a phytoconstituent obtained from the aerial portions of a Chinese medicinal herb qing hao (Artemisia annua L., annual or sweet wormwood). Decoctions of this herb have been used by Chinese herbalists for more than 1,000 years in the treatment of many illnesses, such as skin diseases and malaria. The antimalarial activity of qing hao was rediscovered in China in 1971, when a low-temperature ethyl ether extraction of the plant produced encouraging results in mice infected with the malaria parasite Plasmodium berghei. In 1972, refinement of the antimalarial fractions led to the isolation of a novel crystalline compound which possessed the principal antimalarial activity [1]. Artemisinin has a completely new antimalarial prototype structure with endoperoxide moiety. Heme iron within the parasite catalyzes the cleavage of the endoperoxide bridge followed by formation of a carboncentered radical that alkylates and damages macromolecules in the parasite. The enzyme sarcoplasmic-endoplasmic reticulum ATPase PfATP6 is critical for the parasite survival which is damaged by carbon-centered free radicals [2]. Since artemisinin itself has poor bioavailability limiting its effectiveness, several semisynthetic derivatives of artemisinin have been developed (fig. 1). The best known among these derivatives are oilsoluble artemether, water-soluble artesunate and artenimol ( $\beta$-dihydroartemisinin).

\section{KARGER}

Fax +4161306 1234 E-Mail karger@karger.ch www.karger.com
Dr. Bikash Medhi

Department of Pharmacology

Postgraduate Institute of Medical Education and Research

Chandigarh 160012 (India)

Tel. +91 172275 5250, Fax +91 172274 4401, E-Mail drbikashus@yahoo.com 


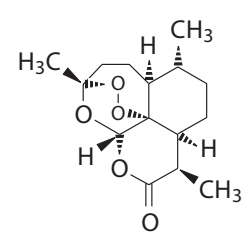

Artemisinin

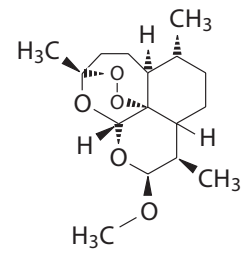

Artemether
Artenimol (dihydroartemisinin)

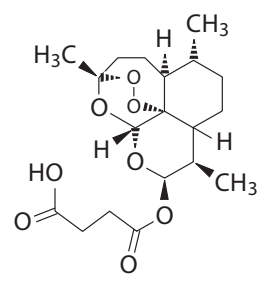

Arteether

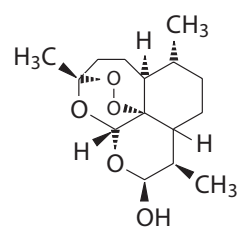

Fig. 1. Structural formulae for artemisinin and its derivatives.

Malaria, a protozoan disease transmitted by the bite of infected anopheles mosquitoes, carries an increased risk of morbidity and mortality. The P. falciparum species is notorious as it can cause severe malaria. If appropriately treated it carries the risk of mortality of $0.1 \%$ but with the involvement of vital organs or $>3 \%$ erythrocyte involvement, the mortality rises steeply. Apart from involvement of cerebrum, renal, pulmonary and coagulation, it can also cause hypoglycemia and hypotension. So, severe falciparum malaria is a medical emergency requiring immediate antimalarial treatment apart from concomitant supportive management.

Chloroquine and sulfadoxine/pyrimethamine are no more used due to drug resistance. Resistance against atovaquone/proguanil and mefloquine has also been documented. Quinine has been used but it has limitations of toxicity and the emergence of drug resistance. Artemisinins are potent and fast-acting antimalarials and can be used in the treatment of severe falciparum malaria. Though these agents are not approved by the FDA, they are used as first-line agents in China and Southeast Asia. They have also gained importance in combination therapy for drug-resistant infections. Rapidly-acting artemisinin compounds are usually combined with mefloquine, amodiaquine, sulfadoxine/pyrimethamine, lumefantrine, piperaquine, pyronaridine, chlorprogua- nil/dapsone. They inhibit gametocyte formation, increase cure rates and delay further transmission of resistant parasites. In this review, we discuss the pharmacokinetics and toxicology of artemisinin and its derivatives $[1,3]$.

\section{Artemisinin Pharmacokinetics}

A large number of comparative studies have been done to determine the kinetic parameters of artenimol, artesunate, artemether, arteether and artelinate in the plasma of rats and those of arteether also in dogs $[4,5]$. A comparison of human data is presented in table 1 [6].

\section{Absorption and Bioavailability}

There are several dosing routes for artemisinin, including i.v., i.m., oral and rectal. Artesunate is suitable for administration through all routes while artemether can be used by i.m., oral or rectal route. All three formulations can be given orally. Oral bioavailability is $30 \%$ due to high first-pass metabolism. While artesunate reaches peak levels within minutes, artemether peaks in 2-6 h. They have modest plasma protein binding ranging from 43 to $81.5 \%$. Both of them are extensively metabolized and converted to dihydroartemisinin which has a plasma half-life of 1-2 h. It accounts for much of antimalarial activity. The other metabolite of artemisinin is artenimol. The water-soluble artesunate gets rapidly converted while the lipid-soluble formulation including artemether is released slowly from the IM site [7]. The rectal administration has emerged as one of the important routes, especially in tropical countries where it can be life-saving. But there is large interindividual variation in the drug bioavailability [8].

\section{Distribution, Metabolism and Excretion}

Tissue distribution of artesunate has been investigated in a study in rats after an i.v. injection of $50 \mathrm{mg} / \mathrm{kg}$. Rats were killed either 10 or 60 min after drug administration, and tissue concentrations were measured by a radioimmunoassay. Shortly after drug administration, the levels in the intestine were markedly higher than in any other organs; brain levels were second, followed by the concentrations in kidney and liver. One hour after the injection, the levels were markedly reduced and were more similar between organs; artesunate concentrations in brain and 
Table 1. Comparative pharmacokinetics of artemisinin derivatives following different routes of administration in healthy and disease conditioned people

\begin{tabular}{|c|c|c|c|c|c|c|c|c|c|c|c|c|c|c|c|c|c|c|c|}
\hline \multirow[t]{2}{*}{ Drugs } & \multirow{2}{*}{$\begin{array}{l}\text { Patient } \\
\text { status }\end{array}$} & \multicolumn{3}{|l|}{$\mathrm{n}$} & \multicolumn{3}{|c|}{ Dose, mg/kg } & \multicolumn{3}{|c|}{$\mathrm{C}_{\max }, \mathrm{ng} / \mathrm{ml}$} & \multicolumn{3}{|c|}{ AUC, $\mu g \cdot h / l$} & \multicolumn{3}{|c|}{$\mathrm{T}_{\max }, \mathrm{h}$} & \multicolumn{3}{|c|}{$\mathrm{T}_{1 / 2}, \mathrm{~h}$} \\
\hline & & OR & IM & $\mathrm{R}$ & OR & IM & IR & OR & IM & $\mathrm{R}$ & OR & IM & $\mathrm{R}$ & OR & IM & $\mathrm{R}$ & OR & IM & $\mathrm{R}$ \\
\hline \multirow[t]{8}{*}{ Artemisinin } & healthy & 10 & 5.3 & 15 & 12 & 10 & 5 & 391 & 209 & 40 & 2,054 & 2,419 & & 1.81 & 3.4 & 4.6 & 2.59 & 7.44 & 4.8 \\
\hline & & 5.3 & & 10 & 10 & & 9 & 260 & & & 819 & & & 1.0 & & & 1.9 & & \\
\hline & & 10 & & 5.3 & 6 & & 10 & 623 & & & 2,611 & & & 2.66 & & & 2.51 & & \\
\hline & & 6.3 & & & 6 & & & 360 & & & 1,190 & & & 1.67 & & & 2.61 & & \\
\hline & & 10 & & & 8 & & & 581 & & & 2,228 & & & 2.5 & & & 2.42 & & \\
\hline & disease & 9.2 & & 10 & 19 & & 6 & 615 & & 180 & 2,234 & & & & & 9.6 & 2.2 & & 4.0 \\
\hline & & 9.2 & & & 19 & & & 588 & & & 2,601 & & & 2.4 & & & 2.3 & & \\
\hline & & 10 & & & 11 & & & 364 & & & & & & 2.88 & & & 2.72 & & \\
\hline \multirow{2}{*}{$\begin{array}{l}\text { Dihydroar- } \\
\text { temisinin }\end{array}$} & healthy & 1.1 & & 8 & 3 & & 5 & 130 & & 100 & & & & 1.33 & & 4.7 & 1.63 & & 4.82 \\
\hline & & 2.2 & & & 3 & & & 710 & & & & & & 1.33 & & & 1.57 & & \\
\hline \multirow[t]{4}{*}{ Artesunate } & healthy & 3.6 & & & 6 & & & 570 & & & 740 & & & $0.5-1$. & & & 0.65 & & \\
\hline & & 2.6 & & & 6 & & & 119 & & & 100 & & & 0.71 & & & 0.27 & & \\
\hline & & 3.5 & & & 1 & & & 577 & & & 312 & & & 0.5 & & & 0.39 & & \\
\hline & disease & 1.64 & 2 & 15.6 & 6 & 6 & 10 & & & 301 & & 510 & 871 & & & 1.83 & & 0.49 & 1.86 \\
\hline \multirow[t]{5}{*}{ Artemether } & healthy & 3.6 & 6 & 5 & 6 & 6 & 8 & 118 & 145 & 75 & 1,100 & 5.2 & 625 & 3 & 7.7 & 3.1 & 3.1 & & \\
\hline & & 5.5 & 10 & & 6 & 6 & & 474 & 224 & & 2,170 & 6.3 & & 2 & 11.1 & & 2.8 & & \\
\hline & & 3.5 & 5.5 & & 6 & 8 & & 310 & 540 & & 671 & 5,200 & & 1.88 & 3.9 & & 2 & 2 & \\
\hline & & 5 & 5 & & 8 & 8 & & 121 & 175 & & 491 & 2,648 & & 1.7 & 8.7 & & 2.6 & & \\
\hline & disease & 3.6 & & & 8 & 10 & & 231 & 40.5 & & 5,800 & 245 & & 3 & 1.33 & & 4.2 & & \\
\hline
\end{tabular}

Patient status: 'healthy' refers to normal people; disease status defined as patients diagnosed with acute, uncomplicated falciparum malaria. $\mathrm{OR}=$ Oral; $\mathrm{IM}=$ intramuscular; $\mathrm{R}=$ rectal; $\mathrm{n}=$ number of patients in the study; $\mathrm{C}_{\max }=$ maximum peak concentration; $\mathrm{T}_{\max }=$ peak time at which $\mathrm{C}_{\max }$ achieved; $\mathrm{AUC}=$ area under curve; $\mathrm{T}_{1 / 2}=$ half life.

fat seemed to be declining at a lower rate than in other organs [9].

Artemisinin and all its derivatives metabolize to form the dihydro-form of artemisinin and artenimol. It was observed that the artemisinin disappearance rate was more rapid in incubations with liver microsomes from rats pretreated with oral artemisinin $(60 \mathrm{mg} / \mathrm{kg} /$ day for 5 days) compared with microsomes from control animals. After fitting a single-pathway Michaelis-Menten saturable elimination model to the concentration-time data of artemisinin incubations by non-linear regression, model parameters were obtained for each animal separately and by pooling data for pretreated and control animals. Parameter estimates (\% coefficient of variation) from fitting the pooled data indicated a 2 -fold increase in $\mathrm{V}_{\max }$ and a 3 -fold decrease in $\mathrm{K}_{\mathrm{m}}$ with microsomes from artemisinin-pretreated animals. Estimates of intrinsic clearance in microsomes from the pretreated animals were 8 -fold higher compared with controls. Thus, artemisinin appears to be a potent autoinducer of drug metabolism in rats as has also been observed in humans. The present findings suggest caution in the interpretation of repeatdose toxicity studies with artemisinin in rat, unless its pharmacokinetics are simultaneously monitored, since during multiple administration, the exposure of the drug will not be constant over time [10].

Furthermore, to investigate the role of increased cytochrome $\mathrm{P}_{450}$ (CYP) $2 \mathrm{~B} 6$ or CYP2C9 activities in artemisinin autoinduction, the effects of multiple-dose artemisinin administration on $S$-mephenytoin $N$-demethylation in healthy subjects were evaluated. These results indicated that artemisinin induces the $\mathrm{N}$-demethylation of $S$-mephenytoin probably by an increased activity of CYP2B6. The autoinduction phenomenon of artemisinin may, therefore, be attributed, at least in part, to induction of CYP2B6, because this is the isozyme primarily involved in its metabolism. In addition, artemisinin alters the disposition of $R$-mephenytoin by an unidentified isozyme [11]. The possibility of artemisinin to induce CYP450 was studied in artemisinin-treated (orally 
for 4 days) and vehicle-treated rats using reverse transcriptase polymerase chain reaction. The effect on enzymatic activities in mouse microsomes from multiple artemisinin administration (intraperitonally) to mice was also studied as well as the effect on the expression in mouse primary hepatocytes and HEK293 cells. Increased CYP2B1 mRNA levels in rats could be seen after artemisinin treatment as well as a weak but reproducible increase in the intensity of CYP1A2 [12]. Administration of artemisinin to mice upregulated hepatic CYP2B10-dependent, and to a lesser extent, CYP2A5dependent enzyme activities. In primary hepatocyte culture, artemisinin significantly increased the CYP2B10 mRNA levels whereas the CYP2A5 mRNA levels were increased to a lesser extent. No significant changes were seen in the levels of other CYP enzymes. Artemisinin was an activator of constitutive androstane receptor but not pregnane X receptor in HEK293 cells. The results demonstrated that the drug exerts its effects on drug metabolism via the constitutive androstane receptor that results in upregulation of genes such as the CYP2B. The weaker upregulation of CYP2A5 might also be constitutive androstane receptor-dependent or alternatively, a consequence of artemisinin toxicity. The results of this study are of importance when predicting potential drug-drug interactions in multidrug therapies with artemisinin [12].

The metabolism of artemisinin is affected by the $\mathrm{pH}$, enzymes in the tissue, blood, liver as well as the route of administration. Food intake has no major effect on artemisinin pharmacokinetics. In addition, studies have demonstrated that artemisinin is cleared by the liver, that this clearance does not depend on liver blood flow (i.e., artemisinin is a so-called low-clearance drug), and that absorption of the drug is not affected by food intake [13]. Liver disease had no effect on the availability and clearance of oral artemisinin, indicating that artemisinin has an intermediate hepatic extraction ratio and that there is no significant first-pass effect [14]. To determine the effect of sex difference in artemisinin metabolism, the pharmacokinetics of the antimalarial compound artemisinin were compared in male and female SpragueDawley rats after single-dose i.v. $(20 \mathrm{mg} / \mathrm{kg})$ or i.p. (50 $\mathrm{mg} / \mathrm{kg}$ ) administration of an emulsion formulation. Plasma clearance of artemisinin was $12.0 \mathrm{l} / \mathrm{h} \cdot \mathrm{kg}$ in the male rat and $10.6 \mathrm{l} / \mathrm{h} \cdot \mathrm{kg}$ in the female rat suggesting high hepatic extraction in combination with erythrocyte uptake or clearance. Artemisinin's half-life was approximately $0.5 \mathrm{~h}$ after both routes of administration in both sexes. Values for plasma clearance and half-lives did not statisti- cally differ between the sexes. After i.p. administration, artemisinin's areas under the plasma concentration-time curve (AUCs) were 2-fold higher in the female compared with male rat. Artemisinin disappearance was 3.9-fold greater in microsomes from male compared with female livers and it was inhibited in male microsomes by goat or rabbit serum containing antibodies against CYP2C11 and CYP3A 2 but not CYP2B1 or CYP2E1. The unbound fraction of artemisinin in plasma was lower in plasma obtained from the male when compared with the female rat. These results interpreted the possibility of a marked sex difference, dependent on the route of administration which should be taken into account in the design and interpretation of toxicological studies of artemisinin in this species [15].

Furthermore, studies were done to investigate whether the decrease in artemisinin bioavailability after repeated oral dosing in humans is the result of increased efflux of artemisinin by P-glycoprotein or decreased membrane transport at the intestinal barrier [16]. The effective jejunal permeability $\left(\mathrm{P}_{\text {eff }}\right)$ of artemisinin was investigated using an in situ rat perfusion model. 54 rats were randomized to one of three treatment arms: no pretreatment, pretreatment with artemisinin emulsion for 5 days $(60 \mathrm{mg} / \mathrm{kg} /$ day, p.o.), or pretreatment with emulsion vehicle for 5 days. The rats within each treatment arm were randomized further to be jejunally perfused with either low $(500 \mathrm{ng} / \mathrm{ml})$ or high $(5,000 \mathrm{ng} / \mathrm{ml})$ artemisinin concentration or low artemisinin concentration plus the P-glycoprotein inhibitor $R, S$-verapamil $(400 \mu \mathrm{g} / \mathrm{ml})$. Perfusate samples were assayed for content of artemisinin, $R, S$-verapamil, and perfusion viability markers. Artemisinin $\mathrm{P}_{\text {eff }}$ was $1.44 \pm 0.38,1.17 \pm 0.32$, and $1.71 \pm 0.29\left(10^{-4} \mathrm{~cm} / \mathrm{s}\right)$ in rats receiving no pretreatment and perfused with low, high, or low artemisinin concentration plus verapamil, respectively. Multiple oral dosing of artemisinin did not affect the jejunal permeability of artemisinin. $R, S$-verapamil $\mathrm{P}_{\text {eff }}$ was similar in artemisinin-pretreated rats $\left(1.09 \pm 0.54 \cdot 10^{-4} \mathrm{~cm} / \mathrm{s}\right)$ and rats pretreated with vehicle only $\left(1.07 \pm 0.37 \cdot 10^{-4}\right.$ $\mathrm{cm} / \mathrm{s})$. Thus it was concluded that the decrease in artemisinin bioavailability after multiple oral dosing in humans is probably not a result of changes in P-glycoprotein expression or general intestinal transport. It seems more likely attributed to increased hepatocellular activity. Moreover, artemisinin exhibits high jejunal permeability and is neither a substrate nor inducer of P-glycoprotein [16]. 


\section{Artemisinin Drug Combinations}

Combination of antimalarial agents has been introduced as a response to widespread drug resistance. The higher number of mutations required to express complete resistance against combinations may retard the further development of resistance. Combination of drugs, especially with the artemisinin group, may offer complete and rapid eradication of the parasite load in symptomatic patients and thus reduce the chance of survival of resistant strains. The advantages of combination therapy should be balanced against the increased chance of drug interactions [17].

During the last decade most of the pharmacokinetic and metabolic pathways of antimalarial drugs have been elucidated, including the role of the cytochrome $\mathrm{P}_{450}$ (CYP) enzyme complex. Change in protein binding is not a significant cause of interactions between antimalarial agents. CYP3A4 and CYP2C19 are frequently involved in the metabolism of antimalarial agents. Quinidine is a potent inhibitor of CYP2D6, but it appears that this enzyme does not mediate the metabolism of any other antimalarial agent. CYP2B6 and CYP3A4 are involved in the metabolism of artemisinin and its derivatives, but further studies may reveal involvement of more enzymes. Artemisinin may induce CYP2C19. Several artemisinin drugs suffer from autoinduction of the first-pass effect, resulting in a decline of bioavailability after repeated doses [17]. The pharmacokinetics of the antimalarial artemisinin exhibited an unusual time dependency during a 7-day oral daily regimen of $500 \mathrm{mg}$ in 10 healthy, male Vietnamese adults. Artemisinin's AUC decreased to $34 \%$ (median) by day 4 with a further decrease by day 7 to only $24 \%$ of values obtained after the first day of administration. In 7 subjects re-studied, after a 2-week washout period, artemisinin's AUCs had almost normalized, demonstrating the reversibility of the time-dependent drug disposition. The results suggested artemisinin exhibits an autoinductive effect on drug metabolism of an unusual magnitude. This partly explains why some patients on standard doses, due to subparasiticidal drug levels toward the end of a standard regimen, do not completely clear parasites. The mechanism of this effect is not yet clear, but the possibility of drug-drug metabolic interactions during combination regimens was implicated [18].

The combination of artemisinin drugs with mefloquine and the fixed combination artemether-lumefantrine have been studied widely. Artemether-lumefantrine is the only fixed, artemisinin-based combination anti- malarial drug which is registered internationally and deployed on a large scale. A multiple cross-over pharmacokinetic study was carried out in 12 healthy volunteers to describe the dose-response relationship between co-administration of fat and relative lumefantrine bioavailability. Results showed that absorption of the hydrophobic lipophilic lumefantrine component varies widely between individuals and is greatly increased by fat co-administration, but patients with acute malaria are frequently nauseated and anorexic, making dietary advice difficult to comply with [19]. A cross-over study conducted in adult volunteers demonstrated that artesunate does not influence atovaquone or proguanil pharmacokinetics and the triple-drug combination of atovaquone, proguanil and artesunate was well tolerated [20]. The co-naphthoquine, a novel antimalarial combination, is an oral fixed combination tablet of the naphthoquine phosphate and artemisinin. Artemisinin is characterized by a rapid onset of schizonticidal action and a short half-life. Parasite clearance is, however, often incomplete when it is employed as a single agent unless high dosages are used over several days, but such a regimen may reduce patient compliance and increase the danger of toxicity. Naphthoquine phosphate, by contrast, has a slower onset of action and a longer half-life, associated with a low recrudescence rate. The two components act synergistically clinically providing more rapid relief of symptoms and a higher cure rate than either component alone [21]. There is no interaction between artesunate and mefloquine with respect to tolerance, efficacy, and pharmacokinetics. Single-dose combination therapy with artemisinin drugs and $15 \mathrm{mg} / \mathrm{kg}$ of mefloquine does not completely prevent parasite recurrence and may not prevent mefloquine resistance [22].

The pharmacokinetic properties of piperaquine have also been characterized recently, revealing that it is a highly lipid-soluble drug with a large volume of distribution at steady state/bioavailability, long elimination half-life and a clearance that is markedly higher in children than in adults. The tolerability, efficacy, pharmacokinetic profile and low cost of piperaquine make it a promising partner drug for artemisinin-based combination therapy [23]. The results obtained from a crossover study have suggested that artemisinin compounds alter the pharmacokinetics of phenytoin [24]. Similarly, results of another cross-over study have suggested that artemisinin compounds alter the pharmacokinetics of carbamazepine with significant change in the bioavailability [25]. 


\section{Special Conditions}

Artemisinin concentrations did not decrease during incubation with erythrocyte ghosts suspended in plasma suggesting that the drug does not bind avidly to red blood cell membranes. There was no decline in concentrations of artemisinin in the presence of carbonic anhydrase. The disappearance of the drug in solutions containing hemoglobin was very rapid and was even more so when the incubation was performed under an argon- instead of oxygen-rich atmosphere. The results suggested that drug blood clearance may be considered for inclusion in a pharmacokinetic model, but does not invalidate in vivo plasma concentration-time data and their relevance for clinical effects. Finally, the enhanced artemisinin disappearance when oxygen tension is low may contribute towards the explanation of the selective toxicity of the endoperoxide drugs to Plasmodium falciparum parasite [26].

Thalassemia is common in Southeast Asia, where artemisinin derivatives are frequently used in the treatment of malaria. It has been previously reported that artemisinin derivatives can be concentrated by uninfected thalassemic erythrocytes in vitro but not by normal erythrocytes. As a follow-up to this report, the antimalarial kinetics of intravascular artesunate $(2.4 \mathrm{mg} / \mathrm{kg}$ b.w.) in 10 persons with normal hemoglobins and in 10 patients with thalassemia ( 2 with $\alpha$-thalassemia type 1-hemoglobin Constant Spring and 8 with $\alpha$-thalassemia type 1- $\alpha$ thalassemia type 2) were studied. Concentrations of intravascular artesunate in plasma peaked in both the normal individuals and the thalassemic individuals $15 \mathrm{~min}$ after injection (the first time point). Plasma drug concentrations at all time intervals, except that at $1 \mathrm{~h}$, were significantly higher in thalassemic subjects than in normal subjects. The AUC was 9-fold higher and the volume of distribution at steady state was 15 -fold lower in thalassemic than in normal subjects. In light of the potential neurotoxicity of artemisinin derivatives, these results suggest that thalassemic subjects may need a drug administration regimen different from that of normal patients [27].

\section{Artemisinin Toxicology}

In vitro, animal, and human clinical studies suggest that currently deployed artemisinins possess neurotoxic potential. Hearing loss, ataxia, and tremor are reported from humans. More recently, also reproductive toxicity, specifically the potential of artemisinins for eliciting teratogenic effects, has received enhanced attention.

\section{Neurotoxicity of Parenteral Artemisinin Derivatives}

Previous animal safety studies with qinghaosu derivatives revealed dose-dependent neurotoxicity with movement disturbances and neuropathic changes in the hindbrain of i.m. treated dogs, rats and monkeys. In a pilot study, $20 \mathrm{mg} / \mathrm{kg} / \mathrm{day}$ of artemether was given i.m. to groups of 3 male Beagle dogs for 5 and 30 days, respectively. Clinical signs of neurotoxicity were noted in some individual dogs from test day 23 onwards. Hematologic findings indicated a hypochromic, microcytic anemia. Microscopic examination demonstrated neuropathic changes only at 30 days, but not at 5 days. The animals had neuronal and secondary axonal damage, most prominent in the cerebellar roof, pontine and vestibular nuclei, and in the raphe/paralemniscal region. The affected neurons showed loss of Nissl substance, cytoplasmic eosinophilia, shrinkage of the nucleus and in advanced stages scavenging by microglia. In a subsequent experiment, artemether was administered to groups of 4 male and 4 female dogs, respectively, at 8 daily doses of $0,20,40$ and $80 \mathrm{mg} / \mathrm{kg}$ i.m., or 0, 50, 150 and $600 \mathrm{mg} / \mathrm{kg}$ p.o. Neurologic signs were seen at high i.m. doses only. In most animals they were inconspicuous and consisted of reduced activity with convulsions seen in single dogs shortly before death. Neuronal damage occurred in all animals at 40 and $80 \mathrm{mg} / \mathrm{kg}$ following i.m. treatment. At $20 \mathrm{mg} / \mathrm{kg}$ minimal effects occurred in $5 / 8$ dogs only, indicating that this level was close to tolerated exposure [28].

Similarly, in all experimental mammals tested (rats, dogs, primates), i.m. injections of the oil-soluble antimalarial artemisinin derivatives artemether and arteether produced an unusual pattern of selective damage to brainstem centers predominantly involved in auditory processing and vestibular reflexes. The neurotoxic potential of parenteral artesunate and artemether was compared in a murine model. Adult Swiss albino mice were assigned randomly to 28-day regimens of i.m. artemether or artesunate in doses ranging from 30 to $100 \mathrm{mg} / \mathrm{kg} / \mathrm{day}$. At $30 \mathrm{mg} / \mathrm{kg} /$ day, no abnormalities were detected with either drug. At $50 \mathrm{mg} / \mathrm{kg} / \mathrm{day}$, abnormalities were observed in 6 of 12 artemether recipients and 2 of 12 artesunate recipients. These were reversible in all but 1 (artemether) mouse. At $100 \mathrm{mg} / \mathrm{kg} / \mathrm{day}, 8$ of 36 artemether recipients, 2 of 36 artesunate recipients, and 1 of 18 control mice died. All but 4 surviving mice in the artemether group (86\%) showed obvious and usually irreversible abnormalities of balance and equilibrium, whereas only 4 artesunate recipients (11\%) exhibited abnormalities, and these were reversible in each case. At this dose the relative 
risk for death or disability was 5.3 for artemether recipients. This data indicated that i.m. artemether is significantly more neurotoxic than i.m. artesunate in this murine model [29].

\section{Neurotoxicity of Oral Artemisinin and Its Derivatives}

Using the adult Swiss albino mice model, the drugs were administered daily for 28 days; the neurotoxic potential of the oral drugs was assessed and compared with the parenteral routes of administration. The dose causing neurotoxicity or death in $50 \%$ of animals $\left(\mathrm{ED}_{50}\right)$ was approximately $300 \mathrm{mg} / \mathrm{kg} / \mathrm{day}$ of oral artemether and artesunate compared to $50 \mathrm{mg} / \mathrm{kg} /$ day of i.m. artemether. Doses of i.m. artemether $>100 \mathrm{mg} / \mathrm{kg} /$ day were uniformly lethal. When oral artemether was given in peanut oil, there was an increase in neurotoxicity and mortality compared with the aqueous suspension, and when the food pellets were coated with artemether in oil, giving relatively constant oral intake, neurotoxicity was further increased; $\mathrm{ED}_{50}=150 \mathrm{mg} / \mathrm{kg} /$ day. This data indicated that once-daily oral administration of artesunate or artemether is relatively safe, presumably because the central nervous system (CNS) is exposed transiently, whereas constant exposure either from depot i.m. injection of oilbased drug, or constant oral intake carries relatively greater neurotoxic potential [30]. No comparable lesions were observed after oral administration, artemether levels in the cerebrospinal fluid were $<10 \%$ of plasma levels. After oral administration, artemether concentrations were considerably lower than after i.m. administration. The concentration of dihydroartemisinin was high on day 1 but almost nil on day 7, indicating its fast inactivation in dogs. Two hours after the 8th oral administration, neither artemether nor dihydroartemisinin were detected in cerebrospinal fluid, which may explain the absence of neurotoxicity in dogs after oral administration of artemether [28].

\section{Genotoxicity, Carcinogenicity and Reproductive Toxicity}

To investigate the genotoxic profile of artemisinin, an in vitro bacterial mutagenicity test and an in vivo bone marrow micronucleus test were conducted in mice. Concentrations of up to $300 \mu \mathrm{g}$ per plate in the bacterial assay, and doses of up to $845.6 \mathrm{mg} / \mathrm{kg}$ in the micronucleus test were shown to be without effect, and the compound can thus be regarded as devoid of genotoxic activity [31]. There is no published data on carcinogenicity studies with artemisinins. Due to the short duration of treatment, and in the absence of any indications of a potential carcinogenic hazard, no such studies have been conducted. Although not yet reported to be a problem in clinical use, embryotoxicity is displayed by the compound class in in vitro and in vivo experimental models, in particular by dihydroartemisinin, the main metabolite of all current clinical artemisinins causing embryo death and some abnormalities in early pregnancy in animals. Embryotoxicity appears to be connected with defective angiogenesis and vasculogenesis in certain stages of embryo development. Dihydroartemisinin has been shown in the rat whole embryo culture to primarily affect primitive red blood cells causing subsequent tissue damage and dysmorphogenesis. This may prevent the use of artemisinin derivatives in malaria during pregnancy, when both mother and fetus are at high risk of death. It has been demonstrated that oral administration of artemisinin can adversely affect post-implantation development and pregnancy in the rat. To evaluate whether artemisinin interferes with developmental outcomes at different periods of pregnancy, Wistar rats were treated by gavage with increasing doses of 7,35 and $70 \mathrm{mg} / \mathrm{kg} /$ day from gestational days $7-13$ or $14-20$. Viable embryos and post-implantation losses, and progestagens and testosterone levels, were monitored in the former treatment group and pregnancy and outcomes data, post-implantation losses and male and female developmental endpoints of the offspring were evaluated in the latter treatment group. Results indicated toxicity for both periods of treatment, with lower sensitivity at later stages of pregnancy. The results showed that dosing with 35 or $75 \mathrm{mg} / \mathrm{kg}$ of artemisinin caused high percentages of post-implantation losses that correlated with a trend to lower maternal progestagens and a significant maternal testosterone decrease [32-34].

Artemether has also been tested in mice and rats by i.m. injection, and dosing in the very early stage had no effect on the development of the fetuses, while treatment at the later stages resulted in incomplete embryonic loss. Artesunate has also been found embryolethal and teratogenic in rats, with the most sensitive days being 10 and 11 postcoitum respectively. In this study, pregnant rats were administered a single oral dose of $17 \mathrm{mg} / \mathrm{kg}$ artesunate on days 10-11 postcoitum and conceptuses were evaluated through day 14 postcoitum. The results indicated that embryonic erythroblasts are the primary target of artesunate toxicity in the rat embryo after in vivo treatment, 
preceding embryolethality and malformations. Furthermore, to test the developmental toxicity in an international conference on harmonization-compliant animal studies, embryofetal development studies were conducted in rats and rabbits treated with artesunate alone or a three-drug combination consisting of chlorproguanil hydrochloride, dapsone, and artesunate in the ratio 1.00: 1.25:2.00. Developmental toxicity seen with a three-drug combination consisting of chlorproguanil hydrochloride, dapsone, and artesunate could be attributed to the administered dose of artesunate. The hallmark effect of artesunate exposure was a dramatic induction of embryo loss, apparent as abortions in rabbits and resorptions in both rats and rabbits. In addition, low incidences of cardiovascular malformations and a syndrome of skeletal defects were induced at or close to embryolethal doses of artesunate in both rats and rabbits. The cardiovascular malformations consisted of ventricular septal and vessel defects. The skeletal syndrome consisted of shortened and/or bent long bones and scapulae, misshapen ribs, cleft sternebrae, and incompletely ossified pelvic bones. These developmental effects were observed largely in the absence of any apparent maternal toxicity. The no or low adverse effect levels were in the range of $5-7 \mathrm{mg} / \mathrm{kg} /$ day artesunate $[35,36]$. Another study comparing the developmental toxicity of structurally related artemisinins, dihydroartemisinin, artemether, and arteether to that of artesunate after oral administration to rats on day 10 postcoitum reported similar developmental toxicity and suggested an artemisinin class effect [37]. A study investigating the pregnancy-related effects of artemether reported no changes in rat estrous cycle. Moreover, the results indicated that the drug did not prevent or prolong the rate of conception or parturition, cause preterm delivery and affect litter size [38]. Encouragingly, no adverse drug-related developmental effects have been observed during treatment of multidrug-resistant $P$. falciparum malaria in pregnant women and no congenital abnormality in any of the newborn children was reported [39]. Pharmacovigilance and further studies of the mechanism of developmental toxicity are ongoing to determine whether rats and rabbits are more sensitive to artemisinins than humans.

\section{Other Toxicity}

Non-clinical studies with other toxicity endpoints (immunotoxicity, phototoxicity, local tolerance) have not been performed.

\section{Toxicokinetics}

Multiple doses of arteether at $25 \mathrm{mg} / \mathrm{kg}$ cause CNS and anorectic toxicities in rats. The same dose of arteether was used to study the toxicokinetics after multiple injections and the pharmacokinetics following single administration. These data suggest that arteether may have altered its distribution and elimination in rats as a result of the systemic toxicity. Analysis of the injection sites showed that 38 and $91 \%$ of the total amount of arteether single dose remained in the muscles at $24 \mathrm{~h}$ (after first injection) and $168 \mathrm{~h}$ (at $24 \mathrm{~h}$ after 7 daily multiple doses), respectively. Fast and slow absorption phases from muscle were seen with $\mathrm{T}_{1 / 2}$ of 0.97 and $26.3 \mathrm{~h}$, respectively. The apparent elimination $\mathrm{T}_{1 / 2}$ of arteether after i.m. injection (13.7 h) was much longer than that after i.v. dosing $(0.67 \mathrm{~h})$ due to the prolonged muscle absorption phase. Acute toxicity data of artemisinin drugs demonstrated that animals receiving a high single arteether dose in sesame oil died between days 5 and 11, similar to artemether. When animals received dihydroartemisinin formulated in 50\% dimethylacetamide/oil, or artesunic acid and artelinic acid in $0.9 \%$ saline vehicle, they died between days 1 and 2. This suggests that delayed-onset toxicity and death in the arteether rats may also be due to slow absorption and prolonged drug exposure. Therefore, multiple i.m. administrations cause anorexia and drug accumulation, possibly affecting the toxicokinetics and efficacy of the drug [40].

Comparative toxicokinetic and hydrolysis studies of i.v. administered two new antimalarial agents, artelinate and artesunate, were performed in malaria-infected rats using three daily equimolar doses. Compared to artesunate, higher drug exposure levels and longer exposure time of artelinate in the rat blood may be the cause of its increased toxicity [41].

\section{Safety Pharmacology}

Artemisinin has been investigated in vitro for its potential to interact with potassium ion currents in guinea pig ventricular myocytes. At concentrations of 5, 50 and $100 \mu \mathrm{mol} / \mathrm{l}$, artemisinin inhibited concentration-dependently the potassium current, and no selectivity could be observed for the inhibition of $\mathrm{I}_{\mathrm{Kr}}$ and $\mathrm{I}_{\mathrm{Ks}}$, respectively. Although inhibition of $I_{\mathrm{Kr}}$ is related to the prolongation of the QT interval in the ECG and is thus considered to be an indication of a certain potential for proarrhythmic effects, the concentration range for, as well as the magni- 
tude of, the blocking effect do not seem to indicate any serious problems for the clinical application [42]. Analogous results have been obtained by measuring the influence of artemisinin on action potentials elicited in isolated C-type nodose ganglion neurons by a patch-clamp technique. Artemisinin was used at concentrations of 10 , 30 and $100 \mu \mathrm{mol} / \mathrm{l}$ and was shown to inhibit depolarizing as well as repolarizing action potentials due to the blockade of $\mathrm{Na}^{+}$and $\mathrm{K}^{+}$ion channels [43]. Both i.m. and oral administration of artemether at high dose levels to groups of 4 male and 4 female dogs was associated with prolongation of mean QT interval of ECG, suggesting slowing of repolarization of the myocardium. Individual data indicated that in 1 of 4 females at $80 \mathrm{mg} / \mathrm{kg}$ i.m. this prolongation was above the $25 \%$ level considered as threshold for concern [28]. An extensive study of the effects of artesunate on the central and peripheral nervous system, the cardiovascular, respiratory and gastrointestinal systems of various animal species (mice, rabbits, guinea pigs, dogs, and monkeys) has been conducted with artesunate. The compound was administered as i.v., i.m. or i.p. injections at doses of up to $640 \mathrm{mg} / \mathrm{kg}$. In general, no relevant effects were noted in these species at doses of up to $100 \mathrm{mg} / \mathrm{kg}$. Mice exhibited some effects on the CNS at doses of $200 \mathrm{mg} / \mathrm{kg}$ and above (sedation, increased barbiturate sleeping time, analgesia), and lowered body temperature was observed in rats at a dose of $450 \mathrm{mg} / \mathrm{kg}$, and in rabbits and dogs at doses of $>160 \mathrm{mg} / \mathrm{kg}$. One anesthetized dog was used to estimate the influence on artesunate on the cardiovascular system and exhibited decreased heart rate and blood pressure, and changes in the ECG, at a dose of $320 \mathrm{mg} / \mathrm{kg}$; respiratory depression occurred in the 1 anesthetized dog used for this part at a dose of $640 \mathrm{mg} / \mathrm{kg}$. In rabbits, respiratory depression was observed at doses of $320 \mathrm{mg} / \mathrm{kg}$ and above, without any changes in the blood gases and blood pH. Finally, an in vitro experiment demonstrated an antagonizing effect on barium-induced contractions of intestinal smooth muscle (concentration not specified), but was without influence on acetylcholine-induced contractions [44].

In summary, the main target organs of toxicity are the hematopoietic system with reversible decreases in the number of reticulocytes as the main effect, the cardiovascular system with prolongation of the QT interval, the gastrointestinal system with loss of appetite and weight loss, the live with some slight changes in hepatic serum enzyme activities and concomitant minor histopathology, and especially the CNS with a very specific pattern of neuronal damage seen in the brainstem. Out of these, neurotoxicity has been regarded as a serious problem, but the extent of this neurotoxicity is dependent on the nature of the compound, on the route of administration, and on the nature of the formulation. In addition, artemisinins are very toxic to the developing embryo, exhibiting a very steep dose-response relationship. Teratogenic effects have not been found in most investigations. However, neurotoxicity is not significant in clinical study data, because it occurs at high doses, when plasma levels of artemisinins (parent compound plus artenimol) remain above a certain threshold level (estimated to be in the order of about $40 \mathrm{ng} / \mathrm{ml}$ ). But doses inducing embryotoxicity and teratogenicity in rats and rabbits are very near, or even below, human therapeutic doses thus as a matter of precaution, the use of artemisinins in the first trimester of pregnancy has been contraindicated.

\section{Conclusion}

Artemisinin and its derivatives are highly potent and superior drugs for the treatment of multidrug-resistant falciparum malaria. They have the fastest parasite reduction ratio, broadest parasite stage specificity and are effective against all species of Plasmodium in humans. Due to their short half-life, artemisinin-based combination therapy is gaining importance in which artemisinin compounds are combined with long-acting antimalarial drugs. A discrepancy prevails with regard to the toxicity and safety of the artemisinin family of antimalarials. Thus, further investigations for the development of nonneurotoxic artemisinins are required.

References

1 Shanks GD: Treatment of falciparum malaria in the age of drug resistance. J Postgrad Med 2006;52:277-280.

2 Vennerstrom JL, Arbe-Barnes S, Brun R, et al: Identification of an antimalarial synthetic trioxolane drug development candidate. Nature 2004;430:900-904.

-3 Gautam A, Ahmed T, Batra V, Paliwal J: Pharmacokinetics and pharmacodynamics of endoperoxide antimalarials. Curr Drug Metab 2009;10:289-306.

4 Benakis A, Schopfer C, Paris M, Plessas CT, Karayannakos PE, Dondas I, Kotsarelis D, Plessas ST, Skalkeas G: Pharmacokinetics of arteether in dog. Eur J Drug Metab Pharmacokinet 1991;16:325-328.

5 Li QG, Peggins JO, Fleckenstein LL, Masonic K, Heiffer MH, Brewer TG: The pharmacokinetics and bioavailability of dihydroartemisinin, arteether, artemether, artesunic acid and artelinic acid in rats. J Pharm Pharmacol 1998;50:173-182. 
6 Kyle DE, Teja-Isavadharm P, Li Q, Leo K: Pharmacokinetics and pharmacodynamics of qinghaosu derivates: how do they impact on the choice of drugs and the dosage regimens? Med Trop 1998;58:38-44.

$\checkmark 7$ Navaratnam V, Mansor SM, Sit NW, et al: Pharmacokinetics of artemisinin-type compounds. Clin Pharmacokinet 2000;39:255270.

$\checkmark 8$ Karunajeewa HA, Manning L, Mueller I, et al: Rectal administration of artemisinin derivatives for the treatment of malaria. JAMA 2007;297:2381-2390.

$\checkmark 9$ Zhao K, Song Z: Distribution and excretion of artesunate in rats. CAMS and PUMC 1989;4:186-188.

10 Gupta S, Svensson US, Ashton M: In vitro evidence for auto-induction of artemisinin metabolism in the rat. Eur J Drug Metab Pharmacokinet 2001;26:173-178.

-11 Simonsson US, Jansson B, Hai TN, et al: Artemisinin autoinduction is caused by involvement of cytochrome $\mathrm{P}_{450} 2 \mathrm{~B} 6$ but not 2C9. Clin Pharmacol Ther 2003;74:32-43.

-12 Simonsson US, Lindell M, Raffalli-Mathieu F, Lannerbro A, Honkakoski P, Lang MA: In vivo and mechanistic evidence of nuclear receptor CAR induction by artemisinin. Eur J Clin Invest 2006;36:647-653.

$\checkmark 13$ Dien TK, de Vries PJ, Khanh NX, et al: Effect of food intake on pharmacokinetics of oral artemisinin in healthy Vietnamese subjects. Antimicrob Agents Chemother 1997;41: 1069-1072.

14 De Vries PJ, Nguyen XK, Tran KD, et al: The pharmacokinetics of a single dose of artemisinin in subjects with liver cirrhosis. Bach Mai-Amsterdam Research Group on Artemisinin. Trop Med Int Health 1997;2:957962.

-15 Ashton M, Johansson L, Thornqvist AS, et al: Quantitative in vivo and in vitro sex differences in artemisinin metabolism in rat. Xe nobiotica 1999;29:195-204.

16 Svensson US, Sandström R, Carlborg O, et al: High in situ rat intestinal permeability of artemisinin unaffected by multiple dosing and with no evidence of P-glycoprotein involvement. Drug Metab Dispos 1999;27:227-232.

- 17 Giao PT, de Vries PJ: Pharmacokinetic interactions of antimalarial agents. Clin Pharmacokinet 2001;40:343-373.

-18 Ashton M, Hai TN, Sy ND: Artemisinin pharmacokinetics is time-dependent during repeated oral administration in healthy male adults. Drug Metab Dispos 1998;26:25-27.

-19 Ashley EA, Stepniewska K, Lindegårdh N, et al: How much fat is necessary to optimize lumefantrine oral bioavailability? Trop Med Int Health 2007;12:195-200.
20 Van Vugt M, Edstein MD, Proux S, et al: Absence of an interaction between artesunate and atovaquone-proguanil. Eur J Clin Pharmacol 1999;55:469-474.

21 Wang JY, Cao WC, Shan CQ, et al: Naphthoquine phosphate and its combination with artemisinine. Acta Trop 2004;89:375-381.

22 Hung Le Q, de Vries PJ, Binh TQ, et al: Artesunate with mefloquine at various intervals for non-severe Plasmodium falciparum malaria. Am J Trop Med Hyg 2004;71:160-166.

23 Davis TM, Hung TY, Sim IK, et al: Piperaquine: a resurgent antimalarial drug. Drugs 2005;65:75-87.

24 Sukhija M, Medhi B, Pandhi P: Effects of artemisinin, artemether, and arteether on the pharmacokinetics of phenytoin. Methods Find Exp Clin Pharmacol 2006;28:89-94.

25 Sukhija M, Medhi B, Pandhi P: Effects of artemisinin, artemether, arteether on the pharmacokinetics of carbamazepine. Pharmacology 2006;76:110-116.

26 Bakhshi HB, Gordi T, Ashton M: In-vitro interaction of artemisinin with intact human erythrocytes, erythrocyte ghosts, haemoglobin and carbonic anhydrase. J Pharm Pharmacol 1997;49:223-226.

27 Ittarat W, Looareesuwan S, Pootrakul P, et al: Effects of $\alpha$-thalassemia on pharmacokinetics of the antimalarial agent artesunate. Antimicrob Agents Chemother 1998;42: 2332-2335.

28 Classen W, Altmann B, Gretener P, et al: Differential effects of orally versus parenterally administered qinghaosu derivative artemether in dogs. Exp Toxicol Pathol 1999;51: 507-516.

29 Nontprasert A, Nosten-Bertrand M, Pukrittayakamee S, Vanijanonta S, Angus BJ, White NJ: Assessment of the neurotoxicity of parenteral artemisinin derivatives in mice. Am J Trop Med Hyg 1998;59:519-522.

30 Nontprasert A, Pukrittayakamee S, NostenBertrand M, Vanijanonta S, White NJ: Studies of the neurotoxicity of oral artemisinin derivatives in mice. Am J Trop Med Hyg 2000;62:409-412.

-31 China Cooperative Research Group: Studies on the toxicity of qinghaosu and its derivatives. J Trad Chin Med 1982;2:31-38.

-32 Boareto AC, Muller JC, Bufalo AC, Botelho GG, de Araujo SL, Foglio MA, de Morais RN, Dalsenter PR: Toxicity of artemisinin (Artemisia annua L.) in two different periods of pregnancy in Wistar rats. Reprod Toxicol 2008;25:239-246.

33 Longo M, Zanoncelli S, Torre PD, Riflettuto M, Cocco F, Pesenti M, Giusti A, Colombo P, Brughera M, Mazué G, Navaratman V, Gomes M, Olliaro P: In vivo and in vitro investigations of the effects of the antimalarial drug dihydroartemisinin (DHA) on rat embryos. Reprod Toxicol 2006;22:797-810.
34 White TE, Bushdid PB, Ritter S, Laffan SB, Clark RL: Artesunate-induced depletion of embryonic erythroblasts precedes embryolethality and teratogenicity in vivo. Birth Defects Res B Dev Reprod Toxicol 2006;77: 413-429.

35 D’Alessandro S, Gelati M, Basilico N, Parati EA, Haynes RK, Taramelli D: Differential effects on angiogenesis of two antimalarial compounds, dihydroartemisinin and artemisone: implications for embryotoxicity. Toxicology 2007;241:66-74.

>36 Clark RL, White TE, Clode A, Gaunt I, Winstanley P, Ward SA: Developmental toxicity of artesunate and an artesunate combination in the rat and rabbit. Birth Defects Res B Dev Reprod Toxicol 2004;71:380-394.

37 Clark RL, Lerman SA, Cox EM, Gristwood WE, White TE: Developmental toxicity of artesunate in the rat: comparison to other artemisinins, comparison of embryotoxicity and kinetics by oral and intravenous routes, and relationship to maternal reticulocyte count. Birth Defects Res B Dev Reprod Toxicol 2008;83:397-406.

$>38$ Ejiofor JI, Kwanashie HO, Anuka JA: Some pregnancy-related effects of artemether in laboratory animals. Pharmacology 2006;77: 166-170.

39 McGready R, Cho T, Cho JJ, Simpson JA, Luxemburger C, Dubowitz L, Looareesuwan S, White NJ, Nosten F: Artemisinin derivatives in the treatment of falciparum malaria in pregnancy. Trans R Soc Trop Med Hyg 1998;92:430-433.

40 Li QG, Brueckner RP, Peggins JO, et al: Arteether toxicokinetics and pharmacokinetics in rats after $25 \mathrm{mg} / \mathrm{kg} /$ day single and multiple doses. Eur J Drug Metab Pharmacokinet 1999;24:213-223.

$>41$ Li Q, Xie LH, Si Y, Wong E, et al: Toxicokinetics and hydrolysis of artelinate and artesunate in malaria-infected rats. Int J Toxicol 2005;24:241-250

42 Yang B-F, Luo D-L, Bao L-H, Zhang Y-C, Wang HZ: Artemisinin blocks activating and slowly activating $\mathrm{K}^{+}$current in guinea pig ventricular myocytes. Acta Pharmacol Sin 1998;19:269-272.

43 Qiao GF, Yang BF, Li WH, Li BY: Effects of artemisinin on action potentials from Ctype nodose ganglion neurons. Acta Pharmacol Sin 2003;24:937-942.

44 Zhao Y: Studies on systemic pharmacological effects of artesunate. J Trop Med Hyg 1985;88:391-396 